\title{
Base Drag Reduction Caused by Riblets on a GAW(2) Airfoil
}

\author{
Channa Raju* and P. R. Viswanath ${ }_{\mathrm{i}}$ National Aerospace \\ Laboratories, \\ Bangalore 560 017. India \\ Nomenclature \\ - $\quad$ base drag coefficient, [ $\left.C_{p \prime r}(r / c)\right]$ \\ - total drag coefficient \\ - $\quad$ pressure coefficient. ( $p$ - $p$,jlq. \\ - $\quad$ - base pressure coefficient, $\left(p_{b}-\right.$ mis \\ airfoil chord \\ riblet height \\ - $\quad=$ local static pressure \\ - $\quad=$ freestream static pressure \\ - $\quad=$ freestream dynamic pressure $=$ trailing-edge thickness \\ friction velocity \\ $=$ distance along the chord \\ $=$ distance normal to tunnel axis $=$ \\ angle of attack
}

(CD61,1r $\sim \mathrm{C}_{D_{\text {sinsisecos }}}$

kinematic viscosity

Introduction

AMONG various methods explored for turbulent drag reduction on aerodynamic surfaces, riblets have beer the most promising.' As much as $4-8 \%$ of viscous drag reduction has been reported for simple two-dimensional configurationsPlastic sheets with symmetric v-grooves (manufactured by the $3 \mathrm{M} \mathrm{Co}$.) have been employed widely in research- Assessment of viscous drag reduction on twodimensional airfoils. both at low and transonic speeds, has been reported as well.' Excellent reviews on the subject covering aspects of drag reduction and flow structure are contained in Refs. 1 and 7.

There have been very few attempts exploring the fuse of giblets in separated flows, either from the point of view of drag reduction or separation control- $-^{\circ}{ }^{\circ}$ Recently. Krishnan et al.' showed that riblets actually increase the base drag (about 8.7 on a long axisymmetric body with a blunt base at low speeds: the base diameter was about four times the boundary -layer thickness ahead of the base corner- They used $3 \mathrm{M}$ riblet sheers and systematically studied the effect of $h+{ }^{`}$ on base pressure. They also speculated that, while riblets caused an increase in the base drag for a large-scale separated flow (like on the axisymmetric blunt base'), the effect could be favorable on an airfoil with a blunt trading edge, which is a case of a smallscale separated flow.

The present investigation was undertaken specifically to assess the effect of $3 \mathrm{M}$ riblets on the base pressure of an airfoil with a blunt trailing edge. Experiments were made at low speeds on a $13.6 \%$ thick GAW(2) airfoil model, which has a trailing-edge thickness ratio of $0.5 \%$. The results show very clearly that the base drag reduction of an engineering value can be achieved for the optimized riblet geometry.

Experiments

\section{Facility and Model}

The experiments were conducted in a $300 \times 1500 \mathrm{ntm}$ boundary-layer tunnel. The GAW(2) airfoil model. with a $r$ of

Received Jan- 25, 1998: revision received July 8. 1998: acceptr] for publication July 15, 1998. Copyright 1998 by the American Institute of Aeronautics and Astronautics, Inc- All rights reserved

*Scientist. Experimental Aerodynamics Division.

tH d. Experimental Aerodynamics Division. Associate $M 10^{1}$

A 1 AA- 
$600 \mathrm{~mm}$ and a span of $300 \mathrm{~mm}$, having a trailing-edge thickness of $3 \mathrm{~mm}$, was mounted vertically in the test section. The , ${ }_{n}$ Odel was instrumented with 38 static pressure taps of o.d. $1.2 \mathrm{~mm} \sim \mathrm{n}$ the upper and lower surfaces. The base pressure was measured and averaged using three ports distributed along the vicin'iy of the midspan of the model.

\section{hfeasurements}

The tests were performed at a freestream velocity of 30 nil s, providing a chord Reynolds number of $\mathrm{f} x 1^{1}$ '. The bound $\mathrm{ar}_{\mathrm{ar}}$ layer on the top and bottom surfaces of the model was tripped at $10 \%$ chord from the leading edge using a sandpaper ${ }_{s} t_{\mathrm{r}} \mathrm{ip}$ (24 grade, 30 mm wide).

Riblet films with a height of 0.076 and $0.152 \mathrm{~mm}$ were used in this work; they were applied between 0.1 and $0.96 \mathrm{c}$ on both the top and bottom surfaces. Streamwise variations of ii' calculated using an integral turbulent boundary-layer code"' for the measured pressure distributions on the airfoil upper surface at $\mathrm{a}=0$ and $6 \mathrm{deg}$ are displayed in Fig. 1. The riblet films with $\mathrm{h}=0.076$ and $0.152 \mathrm{~mm}$ appear optimum at $\mathrm{a}=\mathrm{f}$ ) and $6 \mathrm{deg}$, respectively, considering viscous drag reduction.

The Freestream dynamic pressure, model surface. and the case pressures were measured using three micromanometers supplied by Furness Controls, UK. The total drag was determined from the picot and static measurements in the wake using the method of Jones," A constant temperature hot-wire anemometer was used to assess the existence of vortex shedding behind the base. Measurements of model static pressures and picot profiles in the wake were made over an angle-ofattack range of -2 to $6 \mathrm{deg}$. The reference configuration for determining drag reduction was the smooth airfoil model without the riblet and with the same transition trip.

Accuracy of the Measured Data

The uncertainties in the measured data estimated using the methodology of Kline and McClintock'" and taking into account repeatability are

$$
\mathrm{AC},=\sim 0.0035 \mathrm{C}, \mathrm{AC},=! 0.015 \mathrm{C}^{\prime \prime}
$$

\section{Two Dimensionality}

The two dimensionality of the flow was assessed by employing the two-dimensional momentum integral in the wake. Picot profiles for the smooth model (without riblets) at three streamwise locations in the wake (xlc $=10,2.5$, and 3.01 were measured for determining the total drag. Excellent constancy of drag coefficient (within the estimated uncertainty) was ob
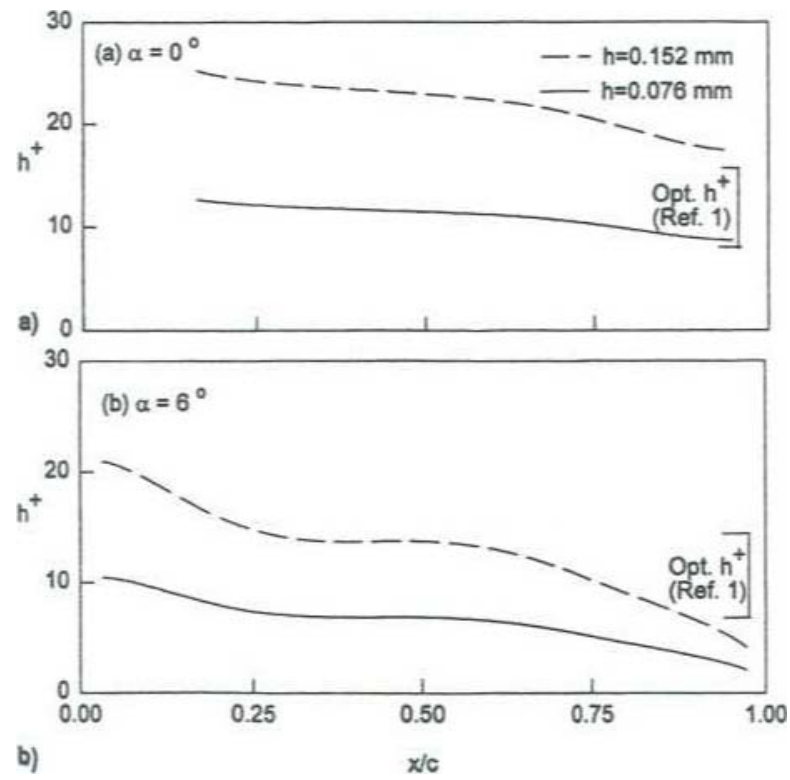

Fig. 1 Variations of h`on upper surface of GAW(2) airfoil. served to suggest good mean flow two dimensionality in the experiments."

\section{Results and Discussions}

Surface Pressure Distributions

The measured surface pressure distributions on the airfoil. both with and without the riblets, revealed" that the effects of riblets on $\mathrm{C}_{\mathrm{A}}$ distributions were very small (as in many earlier studies"'), which suggests that the pressure drag is virtually unaltered because of riblets.

\section{Base Pressure and Base Drag}

The base pressure coefficient for the basic airfoil (without riblets) is positive at all a, indicating a base thrust (Fig_ 2). It is interesting to note that the base pressure progressively increases with riblet height in the a range considered. These results are in contrast with those measured on an axisymmetric blunt base at low speeds." As may be expected, the base drag coefficient is obviously negative because of base thrust, and its magnitude increases further with riblet height. The ratio of

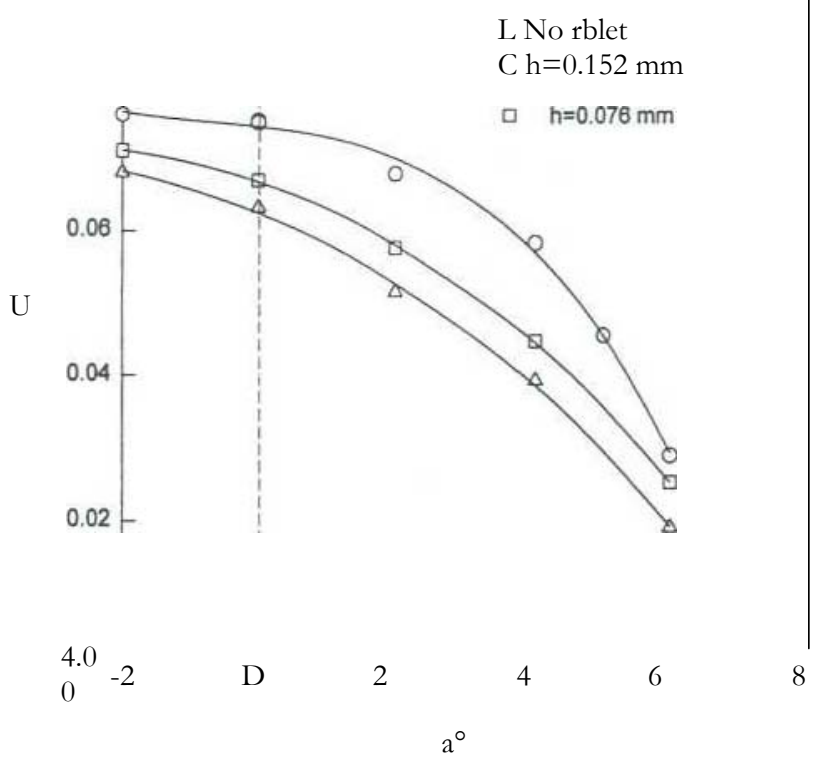

Fig. 2 Variation of base pressure coefficient with incidence.

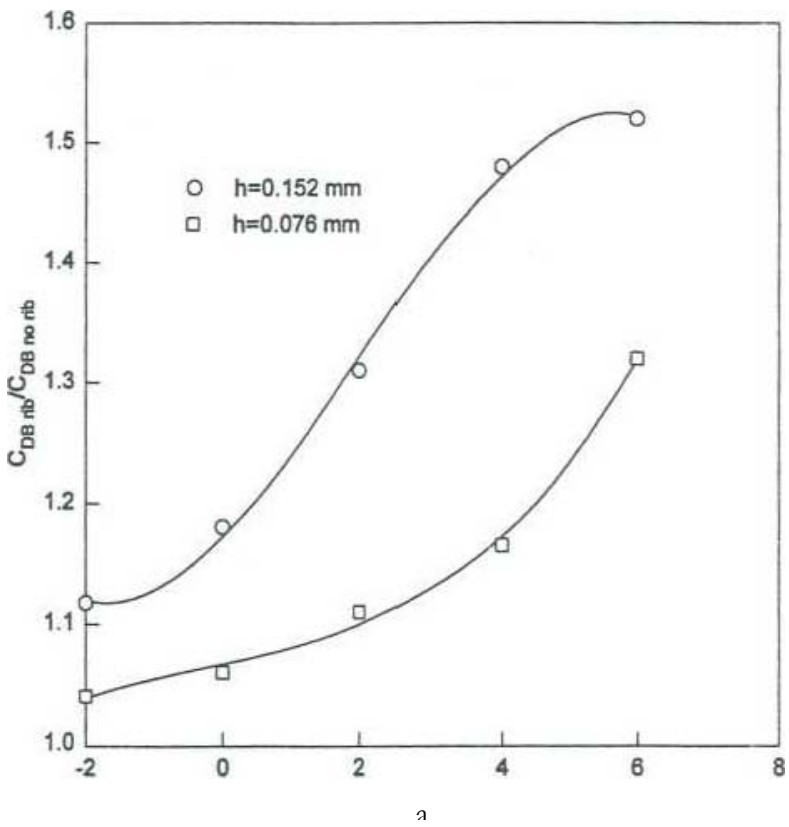

Fig. 3 Variation of normalized base drag with incidence. 


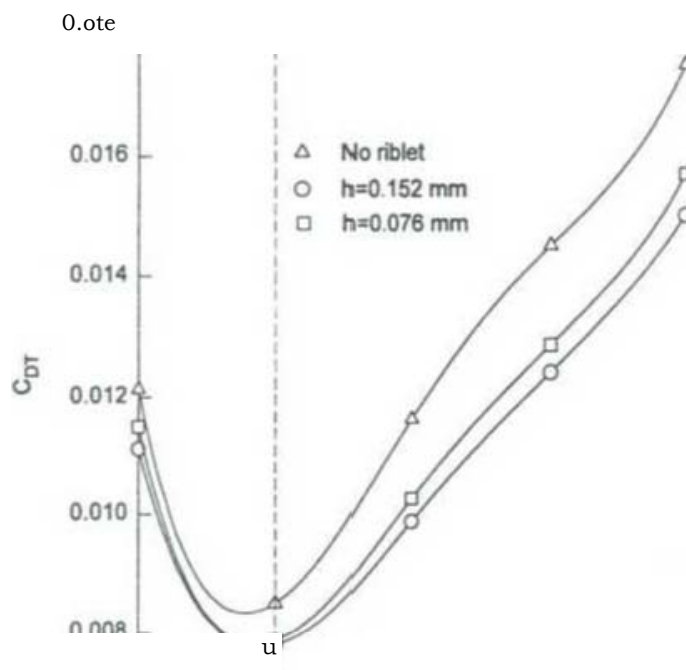

0.006 2 4 $\underline{\mathrm{t}}$ 4 $a^{\circ}$

Fig. 4 Variation of total drag coefficient with incidence.

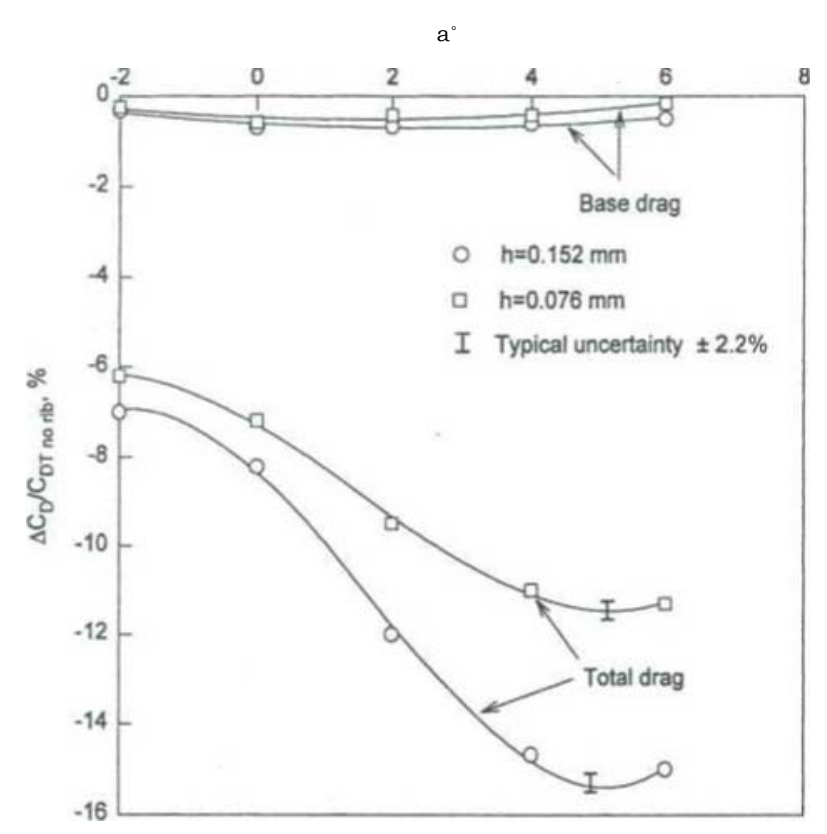

Fig. 5 Total drag and base drag reductions with incidence.

base drag coefficient with riblets relative to no riblets is shown plotted in Fig. 3. The increase in base thrust is as high as 5090 at a $=6 \mathrm{deg}$ for the riblet height of $0.152 \mathrm{~mm}$. The effectiveness of riblet films with $\mathrm{h}>0.152 \mathrm{~mm}$ could not be assessed because they are not manufactured currently by $3 \mathrm{M}$ Co.

\section{Total Drag}

Results of measured total drag coefficient $\left(C_{\text {, }}\right.$ r), both with and without rib€ets, are plotted against airfoil angle of attack in Fig. 4. The riblet film with a height of $0.152 \mathrm{~mm}$ has the lowest drag consistent with the optimum h' variation (discussed in Fig. 1). Figure 5 displays the results of percentage total drag reduction as well as base drag reduction (relative to the smooth baseline configuration); the normalizing factor for both total and base drag reduction is the total drag coeffcient of the smooth airfoil at each a. The increasing trend of total drag reduction with a is a feature already observed by Sundaram et al.' and Subaschandar et al., s' and has been attfibuted to the increased effectiveness of riblets in adverse pressure gradients. The maximum base drag reduction (equivalently an increase in base Ihmst). of about $0.7 \%$ of the total drag ob_ served fair $\mathrm{h}=0_{-} 152 \mathrm{~mm}$, is nearly constant with re.

\section{Possible Flow Mechanisms}

Having observed the increase in base pressure becaus $\mathrm{e}_{\mathrm{e}}$ a riblets, it is appropriate to speculate on possible flow mech $_{\mathrm{a}}$. nisms that may he responsible for the same. Measurement, using a hot-wire probe in the near-wake showed no evidence, of vortex shedding For the baseline as well as the ribbed airfoil configurations, suggesting that the increased base pressure is obviously caused by mean Flow changes because of riblets, $h$ is well known, e.g., Refs. I, 3, and 7 . that riblets lead to lowe $e_{r}$ boundary-layer displacement thickness $\left(\mathbf{S}^{*}\right)$ and, therefore. the effective base height (including $\mathrm{u}^{*}$ effect) is smaller cornpared with the smooth airfoil. and an increase in base pressure eaa be expected." In the context of base fow dynamics. it isgererally known' -" that the base pressure depends on the de velopinent of the free shear layer, which in turn depends as the initial boundary-layer conditions just ahead of the bast; Earlier studies" revealed that the near-wall flow is strongiv affected by riblets, which includes a reduction in turbulent $\mathrm{i}_{-}$- tensities (as much as $10-20 \%$ )' '-'" and Reynolds shear stre.s. e.g., about $15 \%$ in the experiments of Walsh" and Suzuki and Kasagi.' It would therefore seem likely that the combination of lower (mean) velocity gradient and reduced levels of turbulent intensities and shear stress in the wall region of the approaching boundary layer (ahead of the base plane) will Cavorably affect the shear-layer development because the mixing zone is relatively short (comparable to the trailing-edge thickness). It is suggested that the increase in base pressure Is primarily influenced by the initial conditions of the boundary layer just ahead of the base because of riblets leading to (eel. atively) lower velocity along the dividing streamline of the shear layer and, hence, a higher base pressure ${ }^{10}$ in the presence of riblets.

\section{Conclusions}

It has been demonstrated for the first time that riblets can also provide a base drag reduction of engineering value $t \bullet n$ a blunt trailing airfoil at low speeds; the results further show that the base drag reduction is maintained up to an airfoil incidence of $6 \mathrm{deg}$. Although the base drag reduction is large (as much as $50 \%$ of the smooth airfoil base drag), its contribution as a fraction of the total drag in only about $0.7 \%$ because the base drag component itself is small on the airfoil. It is suggested that the increase in base pressure is a direct consequence of certain favorable changes in the boundary layer as a result of riblets ahead of separation; these include a lower effective base height of the airfoil (including boundary-layer displacement thickness) and reduced mixing in the free shear layer leading to lower velocity along the dividing streamline. It would be very informative and valuable to assess base drag reduction because of riblets on supercritical airfoils with a blunt trailing edge at transonic speeds, as well as to investigate, in d'taii. flow mechanisms responsible for the base pressure increase with these riblets_

\section{References}

'Walsh, ht. 1., "giblets." / iscous Drag Reduction in Bowfdan' Layers, edited by D. M. Bushnell and J. N. Hefner. Vol. 123. Progress in Astronautics and Aeronautics, AIAA, Washington. DC, 199.3. pg• 203261.

${ }^{2}$ Coustols. E.. "Control of Turbulence by Internal and External Manipulators," 4rh Internarionrzf Conference on Drag Reduction. edited by A. M. Savill. Vol. 46. No. 3, Applied Scientific Research. K1 uwrr Academic. Norwell, MA, 1999, pp. $183-196$.

'Sundaram, S., Viswanath. P R., and Rudra Kumar, S.. " " a"U Drag Reduction Using Rihlcts on a NACA 0012 Aerofoil Up IC '4'lr'derate Incidence," AMA Journal, Vol. 34, No. 4. 1996. pp- 676-632

'Viswanath, P R., and Mukund. R.. "Turbulent Drag Reu1LcO-' Using Riblets on a Supercritical Airfoil at Transonic Speeds.' $441^{44}$ Journal, Vol. 33. No, 5. 1995. pp. 945-947. 

${ }^{5} 5$ ubaschandar, N., Rajecv Kumar. and Sundaram, S., "Studies of
Turbulent Drag Reduction Due to Riblets on GAW(2) Airfoil," National Aerospace Labs., Rep[. PD EA 9601, March 1996.

s5ubaschandar, N.. Rajeev Kumar, and Sundaram, S., "Drag geduction Due to Riblets on NACA 0012 Airfoil at Higher Angles of Attack," National Aerospace Labs., Rept. PD EA 9504, March 1995

Coustols. E., and Savill. A. M., "Turbulent Skin Friction Reducnon by Active and Passive Means," AGARD Rept., No. 786, FDPI VKI Special Course on Skin Friction Reduction, Belgium, 1992

'Krishnan, V., Viswanath, P. R., and Rudra Kumar, S., - Effect of Riblets on Axisymmetric Base Pressure," Journal of Spacecraft and Rockers, Vol. 34, No. 2, 1997, pp. 256-258.

'King, P. I. Martens, N. W., and Franke, M. E., "Effect of Riblets of Flow Separation in a Subsonic Diffuser," ASME Forum on Tur6ulenr Flovss-1989edited by W. Bower and M. 1. Moms, American Society of Mechanical Engineers, New York, 1989, pp. 79-83.

"Desai, S. S., and Kiske, S.. "A Computer Program to Calculate Turbulent Boundary Layer and Wake in Compressible Flow with Arhitrary Pressure Gradient Based on Greens Lag-Entramment dethod," Bericht No. 8911982Rhur Univ., Bcchum, Germany, 1982.

5chlichting, H., Boundary Layer Theory, 7thed,, McGraw- 14 i 11, New York. 1979.

Kline, S. J., and McClintock, F. A., "Describing Uncertainties in Single Sample Experiments," Mechanical Engineering, Vol. 75, No.
1, 1953, pp. 3-8

"Chana Raju, and Viswanath. P R., "Base Drag Reduction Due to Riblets 1997 .

"Nash, 7. F., "A Discussion of Two-Dimensional Tur ulent 13ase Flows." Aeronautical Research Council, Research Memo unda 3468. London, UK, June 1965.

${ }^{5}$ Chapman, D. R., "An Analysis of Base Pressure at Supersonic Velocities d Comparison with Experiment," NACA, Rept. 1051, Jan. 1951

"Chapman, D. R, Kuehn, D. M , and Larson, H. K, "Investigation of Separated Flows in Supersonic and Subsonic Streams with Emphasis on the Effect of Transition," NACA. Rept. R-1356, 1958.

"Korst, H. H., "A Theory of Base Pressure in Transonic and Supersonic Flow," Journal of Applied Mechanics, Vol. 23, Dec. 1956. pp_ g593600.

Walsh, M. J., "Drag Characteristics of V-Groove and Transverse Curvature Riblets," Yrscous Drag Reduction, edited by G. R. Hough, Vol. 72, Progress in Astronautics and Aeronautics, AIAA, New York, 1980, pp.

Suzuki, Y. and Kasagi. N., "Drag Reduction Mechanism on MicroGrooved Riblet Surface," Proceedings of International Conference an B. E. Launder, Elsevier, Amsterdam, The Netherlands, 1993, pp. 709--7188
Aerothermod namics of

\section{0rm}

Rocket Propulsion Third E d it i o n Gordon C. Dates

AIM textbook

1997, 452 pp, Hardcover

1SEIN 1-56347.241-4 List

Price; $\$ 69-95$

AIAA Member Price: 149.95

Includes Software

Call 800/682-AIAA

\section{Order Today!}

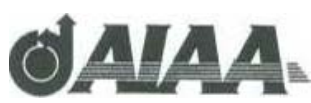

American Institute of Aeronautics and Astronautics PIldii,2t17R5 Custom pr Service 9 Jay Gnuld CI.

PD. Box W

Wa'dA F..ID

$2 \mathrm{U} 604$ rax

301?943-0159
This serninal book on gas turbine technology has been a best-seller since it was first published in 1 984- It now includes a comprehensive set of software programs that complement the text with problems and design analyses- Software topics include: Atmosphere Programs. Quasi-oneDimensional Flows Programs (Ideal Constant-Area Heat Interaction, Adiabatic; Constant-Area Flow with Friction, Rocket Nozzle Performance. Normal Shock Waves, Oblique Shock Waves], Gas Turbine Programs (Engine Cycle Analysis and Engine GffDesign Performance), and Rocket Combustion Programs ( $T$, and $P$, given. $H_{e}$ and $P_{n}$ given, Isentropic Expansion]. Used as a standard text in mare than 50 universities, the book and software will continue to fulfill the need for a comprehensive modern book on the principles of propulsion.

Contents:

Thermodynamics and Duasi-OneDimensional Fluid Flows • Chemical Rockets

- Nonchemicel Rockets s Ideal Cycle Analysis • Component Performance

- Nonideal Cycle Analysis • Engine Off-Design Performance • Elementary Theory of Blade Aerodynamics • Throughft w Theory • Cascade Flows

System Requirements:

RaM • hard drive with 1-17 MS of available disk

wimmm. a i a a, or 


\section{Journal of Aircraft Index}

\section{How to Use the Index}

In the Subject Index, pages 993-998, each technical paper is listed under a maximum of three appropriate headings. Note the number in boldface type following each paper title, and use that number to locate the paper in the Chronological Index. The Author Index, pages 999-1000, lists all authors associated with a given technical paper. The locating numbers are identical to those in the Subject Index. The Chronological Index, pages 1001-1006, lists ail papers by their unique code numbers. This listing contains titles, authors and their affiliations, and volume, issue number, and page where the paper appeared. It also gives the AIAA paper number, if any, on which the article was based, as well as the "CP" or conference volume number il'the paper was published in a hound collection of meetings papers. Comments. Replies, and Errata are listed directly beneath the paper to which they refer. If the paper to which they refer was published prior to 1998, that paper also will appear in the Chronological Index. Authors cat Comments also are listed in the Author Index. 